\title{
Icariin suppresses cell cycle transition and cell migration in ovarian cancer cells
}

\author{
PENGZHEN WANG $^{1}$, JINLI ZHANG $^{1}$, XIFENG XIONG $^{1}$, WEI YUAN ${ }^{2}$, SHENGNAN QIN $^{1}$, \\ WENJUAN CAO ${ }^{1}$, LIBING DAI ${ }^{1}$, FUQIN XIE ${ }^{1}$, AIGUO LI $^{1}$ and ZHIHE LIU ${ }^{1}$ \\ ${ }^{1}$ Guangzhou Institute of Traumatic Surgery; ${ }^{2}$ Department of Surgery, Guangzhou Red Cross Hospital, \\ Jinan University School of Medicine, Guangzhou, Guangdong 510632, P.R. China
}

Received July 26, 2018; Accepted January 22, 2019

DOI: $10.3892 /$ or.2019.6986

\begin{abstract}
Ovarian cancer is the third most common type of gynecological tumor, in addition to being the most lethal. Cytoreductive surgery with chemotherapy is the standard treatment for ovarian cancer. It is necessary to identify novel chemotherapeutic methods, since current chemotherapy treatments are rarely effective for patients with advanced-stage or recurrent ovarian cancer and may cause acute systemic toxicity. Icariin (ICA) is a prenylated flavonol glycoside derived from Herba Epimedii, a medicinal plant with a variety of pharmacological activities, including anticancer, antidiabetic and anti-obesity effects. By analyzing cell viability, cell cycle and cell migration, the present study demonstrated that ICA inhibited the cell viability of the ovarian cancer cell line, SKOV3, and blocked cell cycle transition. ICA inhibited the expression of fuse binding protein 1 (FBP1), a critical regulator of proliferation and tumorigenesis through binding to the $\mathrm{c}-\mathrm{Myc}$ promoter, as well as $\beta$-catenin, a key regulator in ovarian cancer initiation, metastasis, chemoresistance and recurrence. Furthermore, it was indicated that ICA inhibited the migration of SKOV3 cells. In accordance with our previous findings on high FBP1 expression in ovarian cancer, FBP1 was a potential target of ICA in ovarian cancer cells. Based on these results, the present study demonstrated that ICA may be a potential therapeutic agent for ovarian cancer treatment.
\end{abstract}

\section{Introduction}

Following cervical and uterine cancer, ovarian cancer ranks third among all types of gynecological tumors, in addition to being the most lethal (1). It is quite difficult to identify the tissue type and whether the tumor is benign or malignant. Therefore, due to occult early ovarian cancer symptoms, $>70 \%$

Correspondence to: $\mathrm{Dr}$ Zhihe Liu, Guangzhou Institute of Traumatic Surgery, Guangzhou Red Cross Hospital, Jinan University School of Medicine, Guangzhou, Guangdong 510220, P.R. China E-mail: zliu0731@163.com

Key words: icariin, ovarian cancer, cell cycle, metastasis of patients with ovarian cancer are diagnosed at an advanced stage, limiting treatment options (2). Cytoreductive surgery with chemotherapy is the standard treatment for ovarian cancer. However, treatments currently available are rarely effective for patients with advanced-stage or recurrent ovarian cancer, and may cause severe systemic toxicity (3). Therefore, it is essential to develop safer and more efficient cancer treatment options. Icariin ( $\mathrm{ICA} ; \mathrm{C}_{33} \mathrm{H}_{40} \mathrm{O}_{15} ; 676.65 \mathrm{~g} / \mathrm{mol}$ ) is a prenylated flavonol glycoside derived from the medical plant Herba Epimedii. Numerous studies have demonstrated that ICA and a number of ICA subtype derivatives, exert a wide range of physiological activities, including antioxidant, anti-inflammatory, antimicrobial, anticancer, antidiabetic and anti-obesity (4). Our previous study showed that ICA promotes articular cartilage repair by activating hypoxia inducible factor 1 subunit $\alpha$ expression (5).

The far upstream element (FUSE)-binding protein 1 (FBP1) has been identified as a proliferative protein that is overexpressed in a number of malignancies, including hepatocellular carcinoma $(6,7)$, non-small cell lung cancer (7) and colorectal cancer (8). The FUSE is a sequence required for the proper expression of the human c-Myc gene, which serves a critical role in proliferation and tumorigenesis (9). It is located $1.5 \mathrm{~kb}$ upstream of the $\mathrm{c}-\mathrm{Myc}$ promoter $\mathrm{P} 1$ and binds the FBP1 (10-12). FBP family members (FBP-1/2/3) are multifunctional factors and have been linked with various transcript-processing steps, including splicing, mRNA stabilization and degradation (13). FBP1 was originally identified as a single-stranded DNA binding protein, binding to FUSE (14). In our previous studies, it was demonstrated that FBP1 expression was higher in ovarian cancer tissues compared with normal ovarian tissues. In addition, the knockdown of FBP1 enhanced the sensitivity of ovarian cancer cells to carboplatin (15). Furthermore, it was indicated that FBP1 was a target of the histone-lysine N-methyltransferase EZH2 inhibitor, GSK343, in osteosarcoma cells (16). In addition, ICA has been reported as an excellent candidate antitumor agent, exhibiting an anticancer therapeutic effect on ovarian cancer cells (17). However, the underlying mechanism of the anticancer effects of ICA on ovarian cancer remain unclear.

Taking into consideration that ICA exerts anticancer activity, and FBP1 has been reported to be overexpressed in ovarian cancer, the aim of the present study was to demonstrate the inhibitory effect of ICA on ovarian cancer and 
examine FBP1 as a potential target of ICA. Furthermore, the influence of ICA on programmed cell death, the cell cycle and the expression of proteins associated with these processes was examined.

\section{Materials and methods}

Cell culture and cell viability assay. Human ovarian cancer cells (SKOV3; cat. no. TCHu185) were obtained from the Type Culture Collection of the Chinese Academy of Sciences (Shanghai, China) and grown in Dulbecco's modified Eagle's medium (DMEM; Gibco; Thermo Fisher Scientific, Inc., Waltham, MA, USA) containing $10 \%(\mathrm{v} / \mathrm{v})$ fetal bovine serum (FBS; Gibco; Thermo Fisher Scientific, Inc.), $2 \mathrm{mM}$ L-glutamine, $100 \mathrm{U} / \mathrm{ml}$ penicillin and $100 \mu \mathrm{g} / \mathrm{ml}$ streptomycin at $37^{\circ} \mathrm{C}$ in a humidified atmosphere with $5 \% \mathrm{CO}_{2}$.

For the cell viability assay, SKOV3 cells were seeded at $4.0 \times 10^{3}$ cells/well on a $96-$ well plate overnight at $37^{\circ} \mathrm{C}$. They were then treated with a range of ICA concentrations $\left(0,10^{-7}\right.$, $10^{-6}$ and $10^{-5} \mathrm{M}$ ) for $48 \mathrm{~h}$. Cell viability was determined by the MTS method in accordance with the CellTiter 96 Aqueous One Solution Viability assay manual (Promega Corporation, Madison, WI, USA). Absorbance was read at $490 \mathrm{~nm}$ with an automated plate reader. The experiment was repeated at least three times.

Colony formation assay. SKOV3 cells (500 cells/well) were plated in 6-well culture plates and cultured in DMEM supplemented with $10 \% \mathrm{FBS}$. After 14 days culture at $37^{\circ} \mathrm{C}$ without (control) or with $10^{-6} \mathrm{M}$ of ICA (ICA-treated), colonies were washed with PBS, fixed with $10 \%$ formalin for $10 \mathrm{~min}$ at room temperature and stained with $0.1 \%(\mathrm{w} / \mathrm{v})$ crystal violet for $10 \mathrm{~min}$ at room temperature. The colonies were counted using an inverted microscope (x200; Ti-U; Nikon Corporation, Tokyo, Japan). Briefly, the cells that formed colonies containing $>50$ cells were considered a colony (5). The colony number of the control group was set as $100 \%$, and the relative number of colonies of the ICA group was the ratio of ICA:control group. The colony formation images were captured by a camera and the experiments were performed in triplicate.

Cell cycle and cell apoptosis analysis. Flow cytometry was used for the analysis of cell cycle distribution and apoptosis. For cell cycle distribution, SKOV3 cells treated with or without $10^{-6} \mathrm{M}$ ICA for $48 \mathrm{~h}$ were collected and fixed in $70 \%(\mathrm{v} / \mathrm{v})$ ethanol overnight at $4^{\circ} \mathrm{C}$, and then incubated with $1 \mathrm{mg} / \mathrm{ml}$ RNase A for $30 \mathrm{~min}$ at $37^{\circ} \mathrm{C}$. Cells were stained with $50 \mu \mathrm{g} / \mathrm{ml}$ propidium iodide [(PI) BD Biosciences, Franklin Lakes, NJ, USA] in PBS containing 1\% Triton X-100 at room temperature for $30 \mathrm{~min}$. The data were acquired using a BD FACScan flow cytometer (FACSAria II; BD Biosciences) and analyzed using BD ModFit LT version 3.3 (BD Biosciences).

For apoptosis, SKOV3 cells which were treated without (control) or with $10^{-6} \mathrm{M}$ of ICA for $48 \mathrm{~h}$ (ICA-treated) were firstly incubated with Annexin V-fluorescein isothiocyanate (FITC) (Beijing Biosea Biotechnology Co., Ltd., Beijing, China) for $30 \mathrm{~min}$ at $4^{\circ} \mathrm{C}$ in the dark, and then incubated with PI $(100 \mu \mathrm{g} / \mathrm{ml})$ for $5 \mathrm{~min}$ at room temperature. Analysis was immediately performed with FACSuite ${ }^{\mathrm{TM}}$ software (BD Biosciences).
Wound healing assay. SKOV3 cells $\left(2 \times 10^{5} / \mathrm{ml}\right)$ were seeded on a 6-well plate and cultured in DMEM containing 10\% FBS. Once cells grew to confluence, an artificial wound was generated by scratching the dish with a sterile P200 pipette tip. The cells were washed twice with PBS to remove cellular debris, and then were cultured for another $48 \mathrm{~h}$ at $37^{\circ} \mathrm{C}$ without or with $10^{-6} \mathrm{M}$ of ICA. The wound edge movements were captured with an inverted phase contrast microscope (x200; Ti-U; Nikon Corporation), and the results were expressed as the distance between the sides of the scratch. The distance in the control group (without ICA) was set to $100 \%$.

Western blot analysis. Western blot analysis was performed according to standard protocols. Whole cell lysates were obtained by lysing SKOV3 cells in radioimmunoprecipitation assay buffer $[150 \mathrm{mmol} / \mathrm{l} \mathrm{NaCl}, 1 \% \mathrm{NP}-40,50 \mathrm{mmol} / \mathrm{l}$ Tris-Cl (pH 8.0), 0.1\% SDS] in the presence of a protease inhibitor cocktail. Protein concentration was determined with a bicinchoninic acid protein assay. Equal amounts of protein (30 $\mu \mathrm{g} / \mathrm{lane})$ separated by SDS-PAGE ( $12 \%$ gel). The proteins were transferred onto polyvinylidene difluoride membranes using a Bio-Rad semi-dry transfer system (Bio-Rad Laboratories, Inc., Hercules, CA, USA). Membranes were blocked with 5\% (w/v) dry milk in Tris-buffered saline with $0.2 \%$ Tween- 20 for $1 \mathrm{~h}$ at room temperature and subsequently incubated overnight with primary antibodies at $4^{\circ} \mathrm{C}$. The primary antibodies used were as follows: Anti-FBP1 (cat. no. sc-136137; 1:500; Santa Cruz Biotechnology, Inc., Dallas, TX, USA), anti- $\beta$-Catenin (cat. no. sc-7963; 1:200; Santa Cruz Biotechnology, Inc.), anti-c-Myc (cat. no. 5605; 1:1,000; Cell Signaling Technology, Inc., Danvers, MA, USA), anti-matrix metalloproteinase (MMP)9 (cat. no. sc-21733; 1:500; Santa Cruz Biotechnology, Inc.), anti-Cyclin D1 (cat. no. sc-2978; 1:200; Santa Cruz Biotechnology, Inc.), anti-Cyclin E (cat. no. sc-2925; 1:200; Santa Cruz Biotechnology, Inc.), anti-p21 (cat. no. sc-2947; 1:200; Santa Cruz Biotechnology, Inc.), anti-microtubule-associated protein $1 \mathrm{~A} / 1 \mathrm{~B}$-light chain 3 [(LC3) cat. no. 2775; 1:1,000; Cell Signaling Technology, Inc.] and anti-GAPDH (cat. no. 5174; 1:1,000; Cell Signaling Technology, Inc.). Membranes were incubated with goat anti-rabbit (cat. no. ARG65351; 1:3,000; Arigo Biolaboratories Corp., Taiwan, China) or goat anti-mouse (cat. no. ARG65350; 1:3,000; Arigo Biolaboratories Corp.) horseradish peroxidase-labeled secondary antibodies for $1 \mathrm{~h}$ at room temperature. The target proteins were detected using an Enhanced Chemiluminescence Western Blotting Substrate kit (cat. no. 32106; Thermo Fisher Scientific, Inc.) and quantified by densitometry using Quantity One 4.6 .7 software (Bio-Rad Laboratories, Inc.).

Reverse transcription-quantitative polymerase chain reaction $(R T-q P C R)$. Total RNA was extracted using TRIzol reagent (Invitrogen; Thermo Fisher Scientific, Inc.) from the SKOV3 cells and converted to cDNA using PrimeScript RT Master mix (Takara Bio, Inc., Otsu, Japan) according to the manufacturer's protocols. qPCR was performed in triplicate using gene-specific primers (Table I) with SYBR-Green labels (Thermo Fisher Scientific, Inc.) according to the providers' protocols. The house-keeping gene GAPDH was used as an internal control, and the abundance of target gene expressions 
Table I. The sequence of primers used for reverse transcription-quantitative polymerase chain reaction.

\begin{tabular}{llc}
\hline Genes & Forward & \multicolumn{1}{c}{ Reverse } \\
\hline FBP1 & 5'-TGATTCCAGCTAGCAAGGCA-3' & 5'-CGGCCCGTCTTGAATCATAA-3' \\
$\beta$-Catenin & 5'-AATGCTTGGTTCACCAGTG-3' & 5'-GGCAGTCTGTCGTAATAGCC-3' \\
Cyclin D1 & 5'-AGCTGTGCATCTACACCGAC-3' & 5'-GAAATCGTGCGGGGTCATTG-3' \\
Cyclin E & 5'-CCATCATGCCGAGGGAGC-3' & 5'-GGTCACGTTTGCCTTCCTCT-3' \\
p21 & 5'-AGTCAGTTCCTTGTGGAGCC-3' & 5'-GCATGGGTTCTGACGGACAT-3' \\
GAPDH & 5'-ACACCCACTCCTCCACCTTT-3' & 5'-TTACTCCTTGGAGGCCATGT-3'
\end{tabular}

FBP1, fuse binding protein 1 .

were normalized against GAPDH. The $2^{-\Delta \Delta \mathrm{Cq}}$ method was used to analyze relative gene expression (18).

Statistical analysis. All data are presented as the mean \pm standard deviation. Student's t-test was used for comparisons between two groups and one-way analysis of variance followed by Fisher's least significant difference was used to analyze differences among multiple groups. All experiments were repeated three times. $\mathrm{P}<0.05$ was considered to indicate a statistically significant difference.

\section{Results}

ICA inhibits the viability and colony formation of SKOV3 cells. The effect of ICA $\left(10^{-7}-10^{-5} \mathrm{M}\right)$ on the viability of SKOV3 cells was assessed. Cell viability was examined using MTS assays. Compared with the control group, $10^{-6}$ and $10^{-5} \mathrm{M}$ of ICA significantly inhibited SKOV3 cell viability $(\mathrm{P}<0.05$; Fig. 1A). ICA inhibited cell viability in a dose-dependent manner. Since there was no significant difference between $10^{-6}$ and $10^{-5} \mathrm{M}$ groups, follow-up experiments were carried out using $10^{-6} \mathrm{M}$ of ICA. The colony formation assays indicated that the number of SKOV cell colonies was less in the group treated with $10^{-6} \mathrm{M}$ of ICA (ICA group) for 14 days, compared with the cells without ICA treatment (Fig. 1B and C). These results revealed that ICA treatment suppressed SKOV3 cell viability.

ICA has no effect on SKOV3 apoptosis or autophagic cell death. In order to elucidate the factors which decreased cell viability, the influence of ICA on programmed cell death, including apoptosis and autophagic cell death, was investigated. Apoptosis was measured with Annexin V-FITC/PI flow cytometry analysis. The apoptotic rate of the control and ICA-treated groups was 5.98 and $5.78 \%$, respectively. ICA treatment did not enhance SKOV3 apoptosis compared with the control SKOV3 cells (Fig. 2A). Among the autophagy-associated proteins, the conversion of LC3-I to LC3-II has been reported to be widely used as a marker of autophagic cell death (19). The present study did not indicate any significant changes in the ratio of LC3-II/LC3-I between control and ICA-treated groups (Fig. 2B and C). These results suggested that ICA treatment did not influence the apoptosis or autophagic cell death of SKOV3 cells.
ICA induces cell cycle arrest and attenuates cycle-associated protein expression in SKOV3 cells. Since ICA had no effect on the apoptosis or autophagic cell death of SKOV3 cells, the cell cycle transition of SKOV3 cells treated with or without ICA was investigated by flow cytometry. A significant elevation of cells in the $G_{1}$ phase and a decline in the $S$ phase was observed in ICA-treated SKOV3 cells (Fig. 3A). ICA treatment increased the percentage of SKOV 3 cells in the $G_{1}$ phase from 60.81 to $66.43 \%(\mathrm{P}<0.05)$, and reduced the percentage of cells in the $\mathrm{S}$ phase from 27.29 to $21.13 \%(\mathrm{P}<0.05)$. The percentage of SKOV3 cells in $\mathrm{G}_{2}$ phase remained virtually unchanged. These data indicated that ICA blocked SKOV3 cell cycle progression at $G_{1}$ phase and inhibited DNA synthesis.

As ICA affected cell cycle pattern, the mRNA and protein expression of cyclins and cyclin-dependent kinase inhibitors (CKIs) was detected in SKOV3 cells treated with and without ICA by RT-qPCR and western blot analysis (Fig. 3B-F). Significant decreases in Cyclin D1 and Cyclin E were observed at the protein and mRNA level in SKOV3 cells treated with ICA (Fig. 3B-E). In contrast, p21 protein and mRNA expression was upregulated in SKOV3 cells treated with ICA (Fig. 3B, C and F). Notably, there was a 39 and 37\% decrease in Cyclin D1 and Cyclin E expression, respectively, accompanied by a $76 \%$ upregulation in $\mathrm{p} 21$ protein expression in response to ICA treatment (Fig. 3B and C). These data revealed that the inhibition of ICA in SKOV3 cell cycle arrest was, at least partially, through the regulation of cell cycle-associated protein expression.

ICA inhibits the expression of FBPI and $\beta$-catenin. FBP1 is known for its regulatory role in c-Myc expression. FBP1 promotes c-Myc expression by binding with the c-Myc promoter $(9,20,21)$. In a previous study, high expression of FBP1 in ovarian cancer tissues was detected $(15,22)$. Therefore, the influence of ICA on FBP1 expression was evaluated in mRNA transcript and protein expression levels. As indicated in Fig. 4A and B, western blot analysis indicated that the expression level of FBP1 protein was downregulated by $45 \%$ by ICA treatment. Furthermore, RT-qPCR indicated a $68 \%$ decrease in FBP1 mRNA expression in response to ICA treatment (Fig. 4C; $\mathrm{P}<0.05$ ). As c-Myc is a direct downstream target of FBP1 and a key mediator of FBP1 signal transduction, the expression of c-Myc was detected by western blotting. 
A

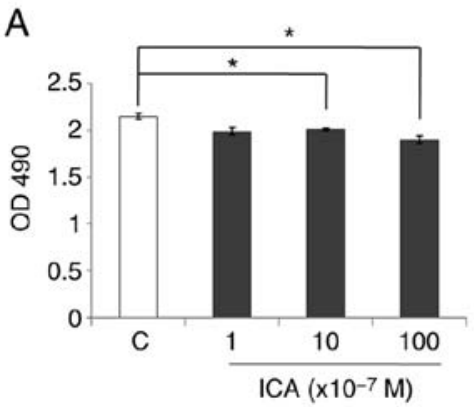

B

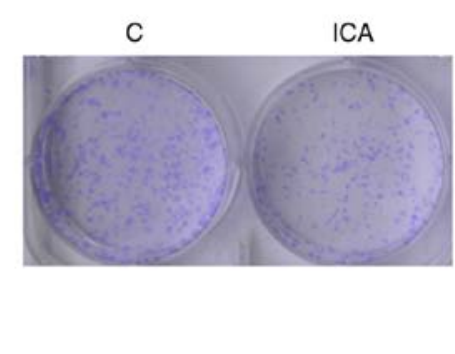

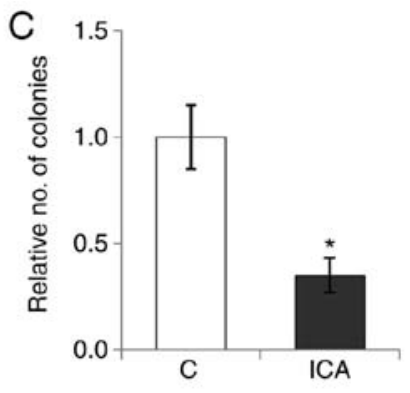

Figure 1. ICA suppresses the viability and colony formation of SKOV3 cells. (A) Cell viability of SKOV3 cells treated for $48 \mathrm{~h}$ with the indicated concentrations of ICA was determined by MTS assays. (B) ICA inhibited colony formation. (C) Relative number of colonies in each group was determined. "P $<0.05$. C, control cells; ICA, icariin-treated cells $\left(10^{-6} \mathrm{M}\right.$ ICA for 2 weeks).

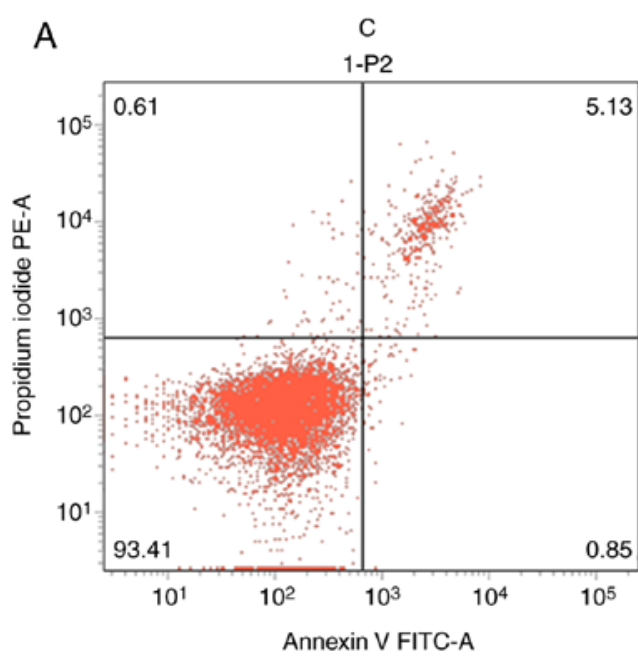

B

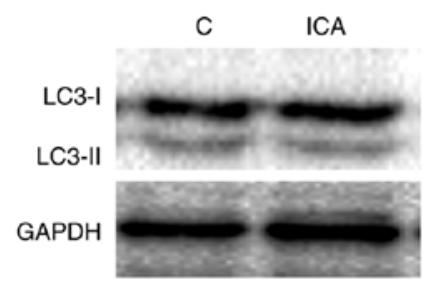

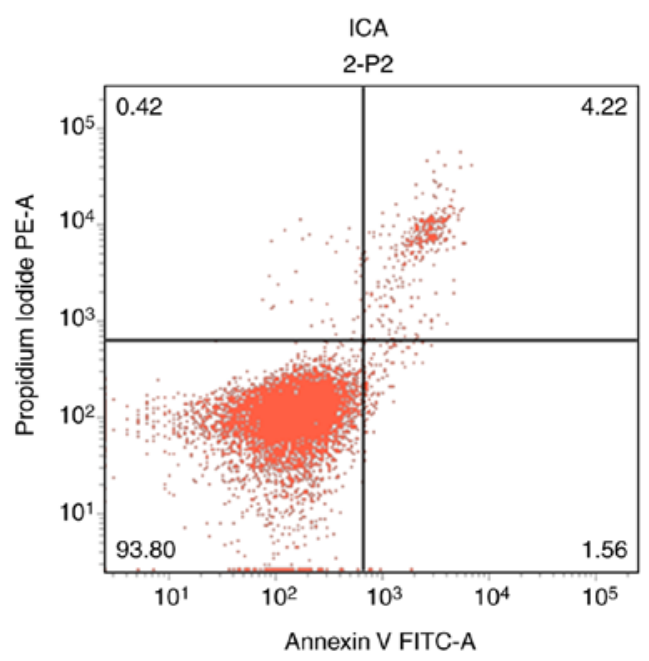

C

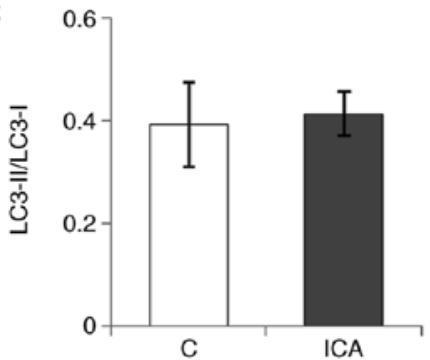

Figure 2. ICA does not influence the apoptosis or autophagic cell death of SKOV3 cells. (A) ICA did not influence apoptosis in SKOV3 cells, as determined by flow cytometry. (B) The expression of autophagy-associated proteins LC3-I and LC3-II in SKOV3 cells was detected by western blotting. GAPDH served as a loading control. (C) The ratio of LC3-II against LC3-I expression. C, control cells; ICA, icariin-treated cells (10 ${ }^{-6} \mathrm{M}$ of ICA for $48 \mathrm{~h}$ ); LC3, microtubule-associated protein 1A/1B-light chain 3.

As shown in Fig. 4E and F, c-Myc protein expression was downregulated by $46 \%$ in the ICA treatment group.

The $\mathrm{WNT} / \beta$-catenin pathway has been reported to contribute to ovarian cancer initiation, metastasis, chemoresistance and recurrence (23). Therefore, the influence of ICA on $\beta$-catenin expression was examined in mRNA transcript and protein expression levels. As demonstrated in Fig. 4A and B, western blot analysis indicated that the expression of $\beta$-catenin protein was downregulated by $39 \%$ by ICA treatment. Furthermore, RT-qPCR indicated a 33\% decrease in $\beta$-catenin mRNA expression with ICA treatment (Fig. 4D; $\mathrm{P}<0.05$ ). These data revealed that the inhibition of SKOV 3 cell viability by ICA was, at least in part, through the regulation of FBP1 and $\mathrm{WNT} / \beta$-catenin expression.
ICA inhibits the migration of SKOV3 cells. Migration is a key property of cancer cells and is required for cancer development (24). Therefore, the influence of ICA on ovarian cancer cell migration was investigated. In the wound-healing assay, the migration distance of ICA-treated SKOV3 cells was significantly lower, compared with the control SKOV3 cells following incubation for $48 \mathrm{~h}(\mathrm{P}<0.05$; Fig. $5 \mathrm{~A}$ and $\mathrm{B})$. There was a 2.1-fold increase in the scratch wound distance of ICA-treated SKOV3 cells compared with the control SKOV3 cells. These results indicated that ICA inhibited the migration of SKOV 3 cells. The MMP protein family, in particular MMP9, serves a very important role in the process of cancer migration and metastasis $(25,26)$. It was indicated that the protein expression of MMP9 in ICA-treated SKOV3 cells was only 
A
C

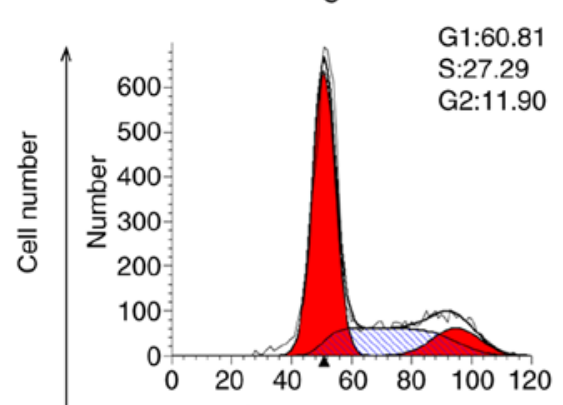

Channels (propidium iodide-apropidium iodide-A)
ICA

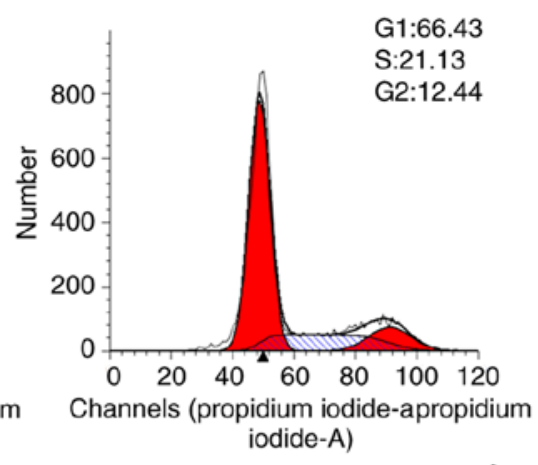

PI

B
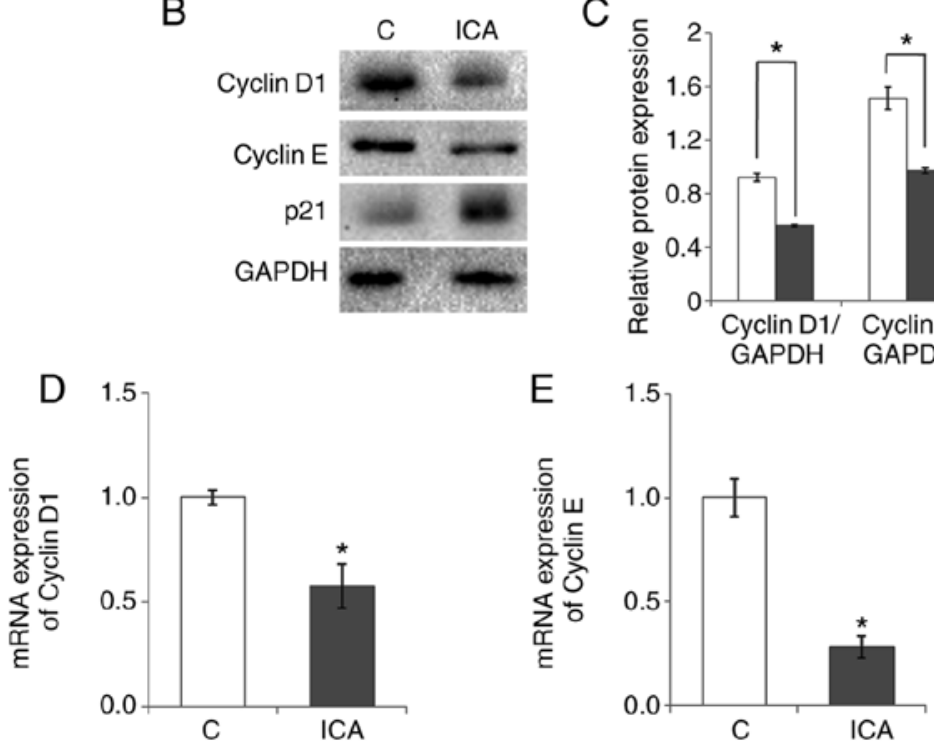

Figure 3. ICA inhibits cell cycle transition and regulates the expression of cell cycle-associated proteins. (A) ICA inhibited cell cycle transition, as determined by flow cytometry. (B) The expression of cell cycle-associated proteins in SKOV3 cells was detected by western blotting and (C) their relative expression was quantified. GAPDH served as a loading control. (D-F) The expression of cell cycle-associated genes (D) Cyclin D, (E) Cyclin E and (F) p21 was measured in SKOV3 cells by reverse transcription-quantitative polymerase chain reaction. ${ }^{*} \mathrm{P}<0.05$. C, control cells; ICA, icariin-treated cells $\left(10^{-6} \mathrm{M}\right.$ of ICA for $\left.48 \mathrm{~h}\right)$; PI, propidium iodide.

A
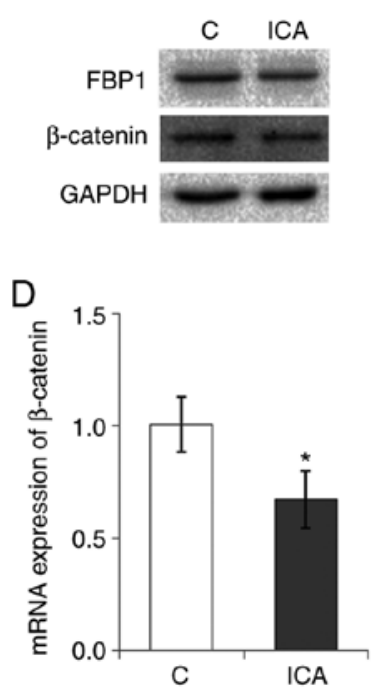

B

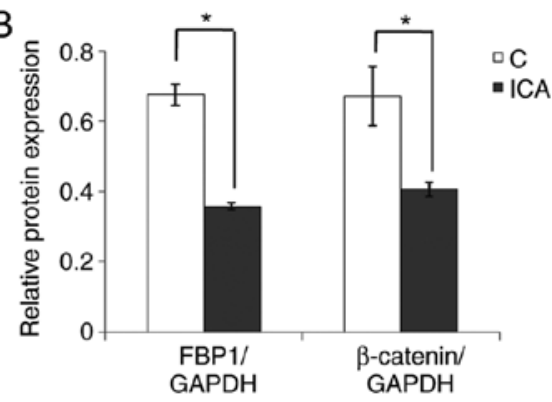

$\mathrm{E}$

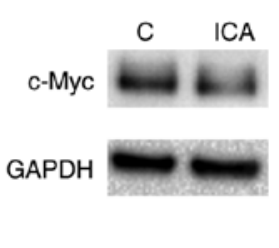

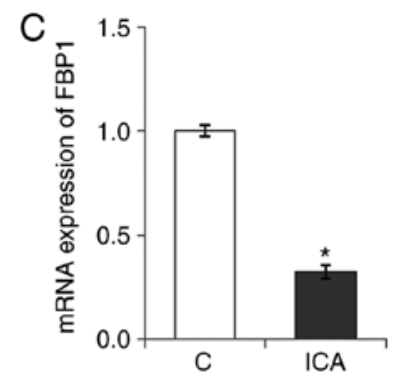

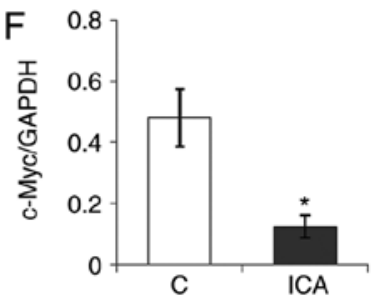

Figure 4. ICA inhibits the expression of proteins associated with cell proliferation. (A) FBP1 and $\beta$-catenin expression in SKOV3 cells was detected by western blotting and (B) their relative expression was quantified. (C) The mRNA expression of FBP1 and (D) $\beta$-catenin in SKOV3 cells was measured reverse transcription-quantitative polymerase chain reaction. (E) c-Myc expression in SKOV3 cells was detected by western blotting (F) and the relative expression of c-Myc was quantified. GAPDH served as a loading control. " $\mathrm{P}<0.05$. C, control; ICA, icariin-treated cells $\left(10^{-6} \mathrm{M}\right.$ of ICA for $\left.48 \mathrm{~h}\right)$; FBP1, fuse binding protein 1 . 
A
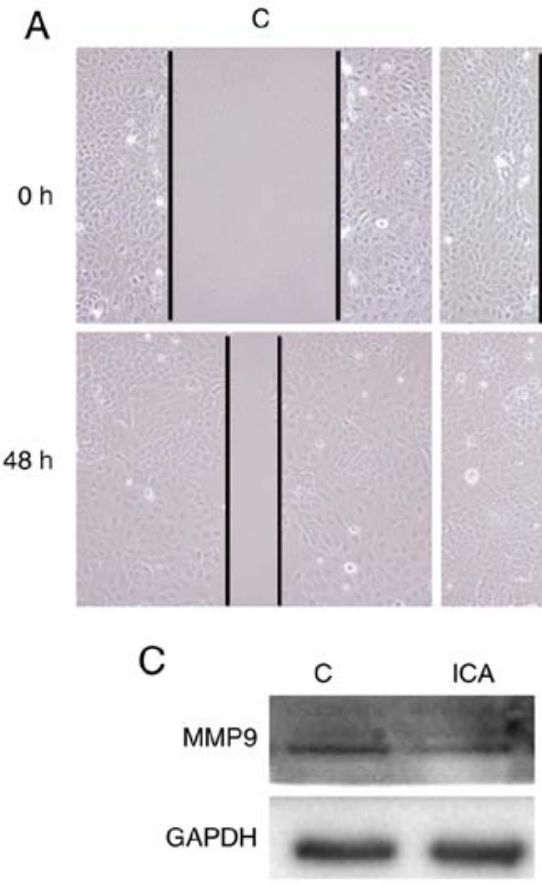

ICA

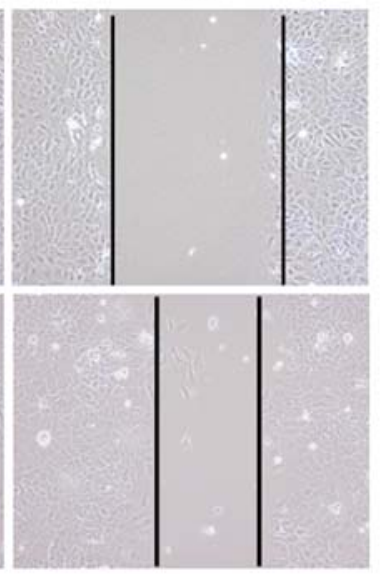

A
B

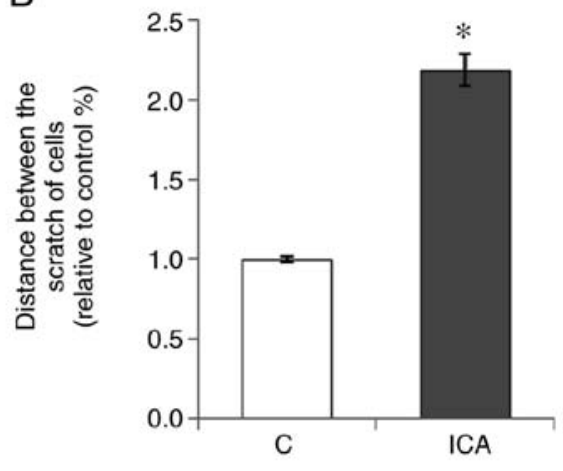

D

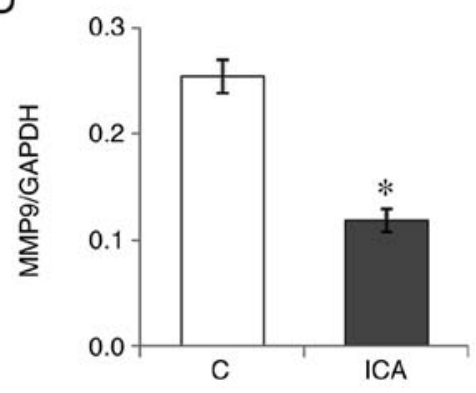

Figure 5. ICA inhibits SKOV3 cell migration. (A) Wound healing assays showed that ICA inhibited cell migration (magnification, x200). (B) The mean distance between the scratch of cells was determined. (C) The expression of MMP9 in SKOV3 cells was determined by western blotting and (D) the relative expression of MMP9 was quantified. GAPDH served as a loading control. " $\mathrm{P}<0.05$. C, control; ICA, icariin-treated cells ( $10^{-6} \mathrm{M}$ of ICA for $\left.48 \mathrm{~h}\right)$; MMP, matrix metalloproteinase.

43\%, compared with the control SKOV3 cells (Fig. 5C and D). These findings demonstrated that ICA exhibited a significant inhibitory effect on the migration of SKOV 3 cells.

\section{Discussion}

Epithelial ovarian cancer is one of the major types of gynecological cancer and has a high mortality rate (27). Although chemotherapy is effective in a number of cases, relapse, drug resistance and toxicity remain a challenge to its therapeutic effectiveness. Thus, the identification of novel potential candidate drugs with less toxic side-effects is essential. It has been reported that ICA has a broad spectrum of anticancer effects, by inhibiting tumor growth (28), tumor cell invasion and migration (29), as well as cell cycle arrest (30).

In the present study, it was demonstrated that ICA inhibited SKOV3 cell proliferation and colony formation. However, no alterations in apoptosis were detected between the control and ICA-treated SKOV3 cells. Furthermore, the LC3-II/LC3-I expression ratio did not show any differences between the control and ICA-treated SKOV3 cells. These findings suggested that ICA did not affect the apoptosis or autophagic cell death of human ovarian cancer SKOV3 cells. This is different with the finding of Li et al (17). Their research indicated that ICA induces the apoptosis of human ovarian cancer cells. This discrepancy may be due to the different concentrations of ICA used in their research $\left(25-50^{-6} \mathrm{M}\right)$, compared to the current study $\left(0.1-10^{-6} \mathrm{M}\right)$.

Cell cycle transition is a promising target for cancer diagnosis and treatment. Deregulation of the $\mathrm{G}_{1}-\mathrm{S}$ phase transition is characteristic in all types of cancer and is the crux of abnormal proliferation (31). Cyclin $\mathrm{E}$ is a well-known Cyclin that regulated the $\mathrm{G}_{1} / \mathrm{S}$ phase transition in combination with cyclin-dependent kinase 2 (CDK2) (32) and Cyclin D1 gene silencing, which also enhances the suppression of chondrocyte proliferation and extends the $\mathrm{G}_{1}$ phase (33). Cyclins and other CDKs, including CDK inhibitor p21, function as the central players of cell cycle regulation, particularly in the transition from $\mathrm{G}_{1}$ to $\mathrm{S}$ phase (34). It has been demonstrated that ICA induces cell cycle arrest at the S phase in A549 cells, and downregulates the expression of $S$ regulatory proteins, including Cyclin A and CDK2 (35). In the present study, the influence of ICA on cell cycle transition was examined and it was shown that ICA treatment induced a higher $\mathrm{G}_{1}$ cell cycle arrest, compared with S-phase in SKOV3 cells. A significant decrease in Cyclin E and D1 expression was observed following ICA treatment. In contrast, p21 expression level was increased with ICA treatment. These results were consistent with previous research, indicating that ICA may inhibit tumor cell proliferation (36). The findings of the present study indicated that ICA may have increased $\mathrm{G}_{1}$ cell cycle arrest and inhibited DNA synthesis in SKOV3 cells.

FBP1 was first identified as a single-stranded DNA binding protein that promotes c-Myc expression (9). In previous studies, it was demonstrated that FBP1 is a key regulator of ovarian cancer development $(15,22)$. In the present study, it was found that ICA treatment reduced the expression of FBP1 and its direct downstream target c-Myc. This result suggested that ICA may have influenced SKOV3 proliferation by targeting the FBP1 signaling pathway. $\beta$-catenin overexpression has been 
reported in epithelial ovarian cancer (37) and may contribute to the carboplatin resistance of ovarian cancer cells (15). In the present study, it was indicated that ICA treatment inhibited the expression of $\beta$-catenin.

Cell migration and invasion are critical factors in the process of tumor metastasis (38). The present study demonstrated that ICA inhibited SKOV3 cell migration. Additionally, ICA treatment decreased the expression of MMP9, a key member of the MMP protein family, which serves an important role in the process of cancer metastasis (26).

In conclusion, the results of the present study demonstrated that ICA reduced the cell viability and colony formation of ovarian cancer cells by inhibiting cell cycle transition and the expression of FBP1 and $\beta$-catenin. In addition, ICA inhibited SKOV3 cell migration. Based on these data, it was hypothesized that ICA may have the potential to inhibit ovarian cancer development, at least partially through the suppression on cell cycle transition and FBP $1 / \beta$-catenin expression. Therefore, ICA is a potential therapeutic agent for ovarian cancer therapy.

\section{Acknowledgements}

The authors would like to thank the staff of Sun Yat-Sen Memorial Hospital, Sun Yat-Sen University (Guangzhou, China) for support with regard to the flow cytometry experiments.

\section{Funding}

The present study was supported by the National Natural Science Foundation of China (no. 81272222 to ZL), the research grants of Guangdong Bureau of Traditional Chinese Medicine (20181206 to ZL), and the Medical Science and Technology Research Foundation of Guangdong (B2016018 and A2018063 to XX), the Guangzhou Science and Technology Program Key Project (201704020145 to SQ).

\section{Availability of data and materials}

All data generated or analyzed during this study are included in this article.

\section{Authors' contributions}

PW and ZL designed the study. PW, JZ, XX, WY, SQ, WC, LD and FX performed the experiments. PW, XX, AL and ZL analyzed the data. PW and ZL wrote the paper. AL contributed in the experimental design and provided general support. All authors read and approved the final manuscript.

\section{Ethics approval and consent to participate}

Not applicable.

\section{Patient consent for publication}

Not applicable.

\section{Competing interests}

The authors declare that they have no competing interests.

\section{References}

1. Luo J, Zhou J, Cheng Q, Zhou C and Ding Z: Role of microRNA-133a in epithelial ovarian cancer pathogenesis and progression. Oncol Lett 7: 1043-1048, 2014.

2. Tang AQ, Cao XC, Tian L, He L and Liu F: Apigenin inhibits the self-renewal capacity of human ovarian cancer SKOV3-derived sphere-forming cells. Mol Med Rep 11: 2221-2226, 2015.

3. Kao YL, Kuo YM, Lee YR, Yang SF, Chen WR and Lee HJ: Apple polyphenol induces cell apoptosis, cell cycle arrest at G2/M phase, and mitotic catastrophe in human bladder transitional carcinoma cells. J Funct Foods 14: 384-394, 2015.

4. Calderón-Montaño JM, Burgos-Morón E, Pérez-Guerrero C and López-Lázaro M: A review on the dietary flavonoid kaempferol. Mini Rev Med Chem 11: 298-344, 2011.

5. Wang P, Zhang F, He Q, Wang J, Shiu HT, Shu Y, Tsang WP, Liang S, Zhao K and Wan C: Flavonoid compound icariin activates hypoxia inducible factor-1 $\alpha$ in chondrocytes and promotes articular cartilage repair. PLoS One 11: e0148372, 2016.

6. Malz M, Weber A, Singer S, Riehmer V, Bissinger M, Riener MO Longerich T, Soll C, Vogel A, Angel P, et al: Overexpression of far upstream element binding proteins: A mechanism regulating proliferation and migration in liver cancer cells. Hepatology 50: 1130-1139, 2009.

7. Rabenhorst U, Beinoraviciute-Kellner R, Brezniceanu ML, Joos S, Devens F, Lichter P, Rieker RJ, Trojan J, Chung HJ, Levens DL, et al: Overexpression of the far upstream element binding protein 1 in hepatocellular carcinoma is required for tumor growth. Hepatology 50: 1121-1129, 2009 .

8. Chen M, Zhang J, Li N, Qian Z, Zhu M, Li Q, Zheng J, Wang X and Shi G: Promoter hypermethylation mediated downregulation of FBP1 in human hepatocellular carcinoma and colon cancer. PLoS One 6: e25564, 2011.

9. Avigan MI, Strober B and Levens D: A far upstream element stimulates c-myc expression in undifferentiated leukemia cells. J Biol Chem 265: 18538-18545, 1990.

10. Duncan R, Bazar L, Michelotti G, Tomonaga T, Krutzsch H, Avigan $M$ and Levens D: A sequence-specific, single-strand binding protein activates the far upstream element of c-myc and defines a new DNA-binding motif. Genes Dev 8: 465-480, 1994.

11. Bazar L, Meighen D, Harris V, Duncan R, Levens D and Avigan M: Targeted melting and binding of a DNA regulatory element by a transactivator of c-myc. J Biol Chem 270: 8241-8248, 1995.

12. Michelotti GA, Michelotti EF, Pullner A, Duncan RC, Eick D and Levens D: Multiple single-stranded cis elements are associated with activated chromatin of the human c-myc gene in vivo. Mol Cell Biol 16: 2656-2669, 1996.

13. Rydziel S, Delany AM and Canalis E: AU-rich elements in the collagenase 3 mRNA mediate stabilization of the transcript by cortisol in osteoblasts. J Biol Chem 279: 5397-5404, 2004.

14. Davis-Smyth T, Duncan RC, Zheng T, Michelotti G and Levens D: The far upstream element-binding proteins comprise an ancient family of single-strand DNA-binding transactivators. J Biol Chem 271: 31679-31687, 1996.

15. Zhang J, Xiong X, Hua X, Cao W, Qin S, Dai L, Liang P, Zhang H and Liu Z: Knockdown of FUSE binding protein 1 enhances the sensitivity of epithelial ovarian cancer cells to carboplatin. Oncol Lett 14: 5819-5824, 2017.

16. Xiong X, Zhang J, Liang W, Cao W, Qin S, Dai L, Ye D and Liu Z: Fuse-binding protein 1 is a target of the EZH2 inhibitor GSK343, in osteosarcoma cells. Int J Oncol 49: 623-628, 2016.

17. Li J, Jiang $\mathrm{K}$ and Zhao F: Icariin regulates the proliferation and apoptosis of human ovarian cancer cells through microRNA-21 by targeting PTEN, RECK and Bcl-2. Oncol Rep 33: 2829-2836, 2015.

18. Livak KJ and Schmittgen TD: Analysis of relative gene expression data using real-time quantitative PCR and the $2^{-\Delta \Delta C_{\mathrm{T}}}$ method. Methods 25: 402-408, 2001.

19. Peng S, Xu LW, Che XY, Xiao QQ, Pu J, Shao Q and He B: Atorvastatin inhibits inflammatory response, attenuates lipid deposition, and improves the stability of vulnerable atherosclerotic plaques by modulating autophagy. Front Pharmacol 9: 438, 2018.

20. He L, Weber A and Levens D: Nuclear targeting determinants of the far upstream element binding protein, a c-myc transcription factor. Nucleic Acids Res 28: 4558-4565, 2000.

21. Liu J, Kouzine F, Nie Z, Chung HJ, Elisha-Feil Z, Weber A, Zhao K and Levens D: The FUSE/FBP/FIR/TFIIH system is a molecular machine programming a pulse of $c-m y c$ expression. EMBO J 25: 2119-2130, 2006. 
22. Xiong X, Zhang J, Hua X, Cao W, Qin S, Dai L, Liu W, Zhang Z, Li X and Liu Z: FBP1 promotes ovarian cancer development through the acceleration of cell cycle transition and metastasis. Oncol Lett 16: 1682-1688, 2018.

23. Arend RC, Londoño-Joshi AI, Samant RS, Li Y, Conner M, Hidalgo B, Alvarez RD, Landen CN, Straughn JM and Buchsbaum DJ: Inhibition of Wnt/ $\beta$-catenin pathway by niclosamide: A therapeutic target for ovarian cancer. Gynecol Oncol 134: 112-120, 2014.

24. Steeg PS and Theodorescu: Metastasis: A therapeutic target for cancer. Nat Clin Pract Oncol 5: 206-219, 2008

25. Liu F, Cao J, Wu J, Sullivan K, Shen J, Ryu B, Xu Z, Wei W and Cui R: Stat3-targeted therapies overcome the acquired resistance to vemurafenib in melanomas. J Invest Dermatol 133: 2041-2049, 2013.

26. Xia Y, Lei Q, Zhu Y, Ye T, Wang N, Li G, Shi X, Shao B, Yin T, Zhao L, et al: SKLB316, a novel small-molecule inhibitor of cell cycle progression, induces G2/M phase arrest and apoptosis in vitro and inhibits tumor growth in vivo. Cancer Lett 355: 297-309, 2014.

27. Li S, Li Y, Wen Z, Kong F, Guan X and Liu W: microRNA-206 overexpression inhibits cellular proliferation and invasion of estrogen receptor $\alpha$-positive ovarian cancer cells. Mol Med Rep 9: 1703-1708, 2014

28. Li S, Dong P, Wang J, Zhang J, Gu J, Wu X, Wu W, Fei X, Zhang Z, Wang Y, et al: Icariin, a natural flavonol glycoside, induces apoptosis in human hepatoma SMMC-7721 cells via a ROS/JNK-dependent mitochondrial pathway. Cancer Lett 298 : 222-230, 2010 .

29. Wang Y, Dong H, Zhu M, Ou Y, Zhang J, Luo H, Luo R, Wu J, Mao M, Liu X, et al: Icariin exterts negative effects on human gastric cancer cell invasion and migration by vasodilator-stimulated phosphoprotein via Racl pathway. Eur J Pharmacol 635: 40-48, 2010.
30. Sun Y, Sun XH, Fan WJ and Li AW: Icariin induces S-phase arrest and apoptosis in medulloblastoma cells. Cell Mol Biol (Noisy-le-grand) 62: 123-129, 2016.

31. Williams GH and Stoeber K: The cell cycle and cancer. J Pathol 226: 352-364, 2012.

32. Odajima J,WillsZP,Ndassa YM,TerunumaM,Kretschmannova K, Deeb TZ, Geng Y, Gawrzak S, Quadros IM, Newman J, et al: Cyclin E constrains Cdk5 activity to regulate synaptic plasticity and memory formation. Dev Cell 21: 655-668, 2011.

33. Zan PF, Yao J, Wu Z, Yang Y, Hu S and Li GD: Cyclin D1 gene silencing promotes IL-1 $\beta$-induced apoptosis in rat chondrocytes. J Cell Biochem 119: 290-299, 2018.

34. Colussi D, Brandi G, Bazzoli F and Ricciardiello L: Molecular pathways involved in colorectal cancer: Implications for disease behavior and prevention. Int J Mol Sci 14: 16365-16385, 2013.

35. Zheng Q, Liu WW, Li B, Chen HJ, Zhu WS, Yang GX, Chen MJ and He GY: Anticancer effect of icaritin on human lung cancer cells through inducing $\mathrm{S}$ phase cell cycle arrest and apoptosis. J Huazhong Univ Sci Technolog Med Sci 34: 497-503, 2014.

36. Ren Y, Zhu F and Liu Z: Inhibitory effect of icariin on osteosarcoma cell proliferation via the $\mathrm{Wnt} / \beta$-catenin signaling pathway. Oncol lett 16: 1405-1410, 2018.

37. Rask K, Nilsson A, Brännström M, Carlsson P, Hellberg P, Janson PO, Hedin L and Sundfeldt K: Wnt-signalling pathway in ovarian epithelial tumours: Increased expression of beta-catenin and GSK3beta. Br J Cancer 89: 1298-1304, 2003.

38. Friedl $\mathrm{P}$ and Wolf $\mathrm{K}$ : Tumour-cell invasion and migration: Diversity and escape mechanisms. Nat Rev Cancer 3: 362-374, 2003. 